Ann. Sci. forest., 1967, 24 (3), 177-203.

\title{
LES SOLS D'UN MASSIF FORESTIER DES BASSES-VOSGES.
}

\section{LES STADES D'ÉVOLUTION}

\author{
F. LE TACON \\ Station de Recherches sur les Sols forestiers et la Fertilisation, \\ Centre national de Recherches forestières, 54 - Nancy \\ Institut national de la Recherche agronomique
}

SOMMAIRE

Au cours de la prospection d'un massif forestier des Basses-Vosges, en vue de l'établissement d'une carte à grande échelle, un certain nombre de problèmes d'ordre génétique se sont posés du fait de la complexité et de l'extrême variété des sols rencontrés sur une surface relativement réduite. II n'était pas possible d'expliquer une telle variété sans faire intervenir des phases de pédogenèses antérieures à l'époque actuelle, ce qui nous a amené à préciser un certain nombre de faits concernant la genèse des sols à horizon de pseudogley, et l'existence de formations rubéfiées.

\section{INTRODUCTION}

Cette étude a débuté par le levé d'une carte des sols à caractères génétiques au $1 / 10000$. Il a été ensuite tiré de ce travail une deuxième carte simplifiée où les sols ont été regroupés et classés uniquement en fonction de leur valeur forestière. Ce travail de cartographie, dont il ne sera pas question ici, a permis de poser un certain nombre de problèmes de genèse du fait de la coexistence de sols peu évolués et de sols très évolués parmi lesquels certains présentent des caractères incompatibles avec le climat actuel. 
Ces notions d'évolution ancienne des sols, au cours des différentes phases climatiques du quaternaire, et même de la fin du tertiaire, sont le résultat de travaux de très nombreux auteurs, notamment en ce qui concerne les sols rubéfiés, dont nous étudierons un exemple. L'hypothèse d'ancienneté de certains sols lessivés, en particulier de sols lessivés à pseudogley, est moins répandue, mais a été émise assez récemment par un certain nombre d'auteurs. Nous nous sommes attachés à démontrer dans ce travail l'intervention du lessivage dans la formation des sols pseudogley de ce massif, et à mettre en évidence le rôle de l'érosion ; ceci nous a amenés à émettre l'hypothèse de l'ancienneté de ces sols, comparativement aux sols bruns et aux sols bruns lessivés, que l'on rencontre parfois dans la même position topographique que les sols lessivés. Nous avons ainsi été amenés à essayer de donner un schéma des différentes phases de pédogenèse qui se sont succédées dans ce massif.

\section{1. - LE MILIEU}

\section{1. - GÉOGRAPHIE}

La forêt de Sainte-Hélène, qui comprend environ un millier d'hectares, est située au Sud-Est de Rambervillers, à cinq kilomètres de cette commune, dans le département des Vosges. Elle est à rattacher à l'ensemble des collines prévosgiennes qui font transition entre la plaine lorraine et le massif montagneux des Vosges. Le climat, dit climat lorrain, est intermédiaire entre celui du bassin parisien, encore sous influence atlantique, et le climat continental d'Europe centrale.

La proximité du massif montagneux des Vosges se traduit par une augmentation de la pluviosité, qui va croissant d'Ouest en Est ( $726 \mathrm{~mm}$ à Nancy, situé à $220 \mathrm{~m}$ d'altitude $-850 \mathrm{~mm}$ à Rambervillers, situé à $290 \mathrm{~m}$ d'altitude), et par un léger abaissement de la température moyenne.

\subsection{Stratigraphie}

Située dans une zone de transition géographique, la forêt de Saint-Hélène s'étend également dans une zone de transition géologique. Du faciès essentiellement détritique du trias inférieur, représenté surtout par des grès, on passe au faciès plus franchement marin du Muschelkalk moyen, essentiellement marneux. En fait, la majeure partie du Muschelkalk et, à un degré moindre, le grès bigarré, sont recouverts par des dépôts récents. Ces dépôts récents consistent en une terrasse alluviale ancienne atteignant une cinquantaine de mètres d'épaisseur, formée de sables grossiers à cailloux roulés, en lits irréguliers souvent entrecroisés. Ces alluvions anciennes sont ellesmêmes recouvertes par des limons, dont on peut estimer l'épaisseur à, au moins, trois mètres.

\subsection{Matériaux originels}

Très rapidement, la prospection a permis de montrer que la plupart des maté- 
riaux originels, sur lesquels s'étaient développés les sols, étaient constitués, non pas par le substratum géologique lui-même, mais par des produits de remaniement, mis en place au quaternaire. Le démantèlement des roches-mères initiales, et l'incorporation les uns aux autres des produits qui en ont résulté, ont abouti à un système complexe de matériaux, qui diffèrent des roches-mères par leur texture et leur compacité.

Au cours des périodes où règnaient des conditions de climat glaciaire ou périglaciaire, les limons ont été affectés par des phénomènes de solifluxion : pendant les phases de dégel, glissement en masse sur les parties profondes constamment gelées ou sur les roches dures sous-jacentes, là où existait une pente légère. Comme les limons reposaient sur des alluvions sableuses à cailloux roulés, la solifluxion s'est traduite par l'incorporation, dans la masse des limons, de cailloux roulés épars. A cette action de la solifluxion s'est ajoutée celle de la cryoturbation qui est un phénomène beaucoup plus localisé, intéressant des poches à l'intérieur desquelles les différents matériaux ont subi des " plications » et des involutions.

Dans les zones à pente plus forte, les limons ont été et sont encore soumis à des phénomènes de remaniement ; les eaux de ruissellement provoquent un déplacement du matériel déjà soliflué en l'ameublissant et en y incorporant plus complètement les éléments grossiers des alluvions sous-jacentes.

Enfin, à la rupture de pente qui est brutale, surtout sur les versants Est et Sud, débutent des phénomènes de colluvionnement caractérisés par des déplacements beaucoup plus rapides, et par un mélange encore plus accentué des limons et des alluvions.

Un quatrième phénomène, que l'on peut distinguer des précédents, consiste en l'entraînement d'éléments fins dans les zones déprimées : cet entraînement aboutit à la formation de limons dits de « colmatage $»$. Ces différentes actions ont permis la différenciation d'une vingtaine de matériaux originels.

\section{2. - LES PRINCIPAUX STADES D'ÉVOLUTION}

La variété des matériaux originels et surtout la diversité de l’âge auquel ces matériaux ont été mis en place, ont permis de conserver les traces de plusieurs pédogenèses différentes.

Nous étudierons successivement par ordre chronologique ces différentes pédogenèses.

Nous débuterons donc par les deux phases de rubéfaction, observées d'une part sur alluvions anciennes, et d'autre part, sur limons anciens, en insistant surtout sur la seconde. Puis nous aborderons la phase de lessivage intense ayant abouti à la différenciation de sols lessivés à pseudogley sur des limons moins anciens.

Enfin, nous terminerons par la phase la plus récente, à laquelle correspondent des sols bruns et bruns lessivés, ainsi que des sols intrazonaux liés à des conditions particulières : podzols et sols à hydromorphie permanente.

Les sols correspondant à cette phase se rencontrent sur les matériaux récemment remaniés ou colluvionnés. 


\section{1. - RUBÉfACtion des alLUVIONS ANCIENNES}

L'ensemble des alluvions anciennes présente une couleur rouge brique constante quelle que soit la profondeur. Les cailloux roulés sont souvent enrobés de pellicules d'oxydes de fer, qui peuvent aussi cimenter entre eux les éléments de toutes les grosseurs. Aucun horizon pédologique proprement dit n'est visible dans les différentes carrières, bien qu'il soit peut-être possible de distinguer à la partie supérieure de ces alluvions une zone un peu plus rubéfiée.

L'absence de véritables horizons, et la présence d'une rubéfaction intense sur plusieurs dizaines de mètres d'épaisseur, semblent indiquer que nous ne sommes pas en présence d'un sol et qu'il n'y a pas eu de pédogenèse en place. La rubéfaction se serait produite antérieurement au dépôt, sur un matériel repris ensuite par un cours d'eau. Rien ne permet de dater ces alluvions. Cependant, en France, les dépôts identiques rubéfiés sont fréquents, et sont habituellement attribués à la fin du pliocène.

\section{2. - SOLS RUBÉFIÉS SUR LIMONS}

Dans une carrière située près de Saint-Gorgon, une poche de limons anciens est bien conservée ainsi qu'un sol fossile à peu près complet, développé à la partie supérieure de ces limons.

Dans ce sol ancien, trois horizons sont visibles : un horizon lessivé II $\mathrm{A}_{2}$, un horizon d'accumulation rouge brique II $\mathrm{B}$ et un horizon IIB/C beige rouge. $\mathrm{L}$ 'horizon organique a disparu et probablement une partie de l'horizon $\mathrm{A}_{2}$.

L'ensemble de ces trois horizons est surmonté d'un colluvium de pente limonosableux dans lequel s'est développé un sol plus récent comprenant un horizon $A_{1}$ et un horizon (B).

\subsection{Description}

\section{A $_{1} \quad$ : Mull Moder \\ $0-5 \mathrm{~cm}$}

(B) : Limon sableux. Très meuble. Structure finement $5-45 \mathrm{~cm}$ grumuleuse. Enracinement très abondant. 10 à $15 \%$ de cailloux roulés. 10 YR 6/4. (Brun jaune clair). Transition brutale avec l'horizon sous-jacent.

Sol brun actuel sur colluvium de pente. 
II $\mathrm{A}_{2}$ : Structure polyédrique très irrégulière, unités de $45-85 \mathrm{~cm} \quad 5$ à $8 \mathrm{~mm}$. Très compact. Nombreux canalicules. Couleur générale 5 YR $5 / 8$ (rouge jaune). $40 \%$ de plages plus claires 5 YR 6/8. Pas de clay-skins. Rares cailloux roulés.

II B : Structure polyédrique à tendance lamellaire. $30 \%$ $85-105 \mathrm{~cm}$ de cailloux présentant une patine ferrugineuse comme les unités structurales. Clay-skins visibles. Rares canalicules. 2,5 YR 4/6 (rouge).

II B/C : Structure polyédrique tendant à devenir nuciforme. $105-140 \mathrm{~cm}$ Quelques enrobements. 1 à $2 \%$ de cailloux roulés. Existence de canalicules. Teinte générale 7,5 YR 5/8 (brun). $30 \%$ de plages 2,5 YR $4 / 8$ (rouge). Transition brutale avec les alluvions.

III C : Alternance de lits sableux et de lits sableux à $70 \%$ de cailloux roulés. 5 YR 5/8 (rouge-jaune).
Sol fossile sur limons anciens

\section{TABLEAU 1}

Caractéristiques analytiques du profil complexe à horizons rubéfiés (carrière de St-Gorgon).

\begin{tabular}{|c|c|c|c|c|c|c|c|c|}
\hline & \multirow{2}{*}{$\begin{array}{l}\mathrm{pH} \\
\text { eau }\end{array}$} & \multicolumn{4}{|c|}{ en $\mathrm{me} / 100 \mathrm{~g}$} & \multirow{2}{*}{$S / T$} & \multirow{2}{*}{$\begin{array}{l}\text { Al libre } \\
\text { (Tamm) } \\
\text { en } \%\end{array}$} & \multirow{2}{*}{$\begin{array}{c}\text { Fe libre } \\
\text { (DEB) } \\
\text { en } \%\end{array}$} \\
\hline & & $T$ & $\mathrm{~K}$ & $\mathrm{Ca}$ & $\mathrm{Mg}$ & & & \\
\hline (B) & 4,4 & 6,25 & 0,15 & 0,05 & 0,02 & 4,0 & 2,7 & 0,92 \\
\hline II $\mathrm{A}_{2}$ & 4,9 & 13,50 & 0,16 & 1,50 & 0,05 & 23,3 & 3,4 & 2,14 \\
\hline II B & 4,9 & 21,40 & 0,16 & 2,65 & 0,06 & 27,6 & 4,6 & 3,76 \\
\hline II B/C & 5,1 & 16,12 & 0,14 & 1,80 & 0,05 & 22,7 & 4,3 & 2,10 \\
\hline \multirow[t]{2}{*}{ III C } & 5,0 & 4,60 & 0,04 & 0,07 & 0,02 & 3,0 & 2,3 & 1,66 \\
\hline & $\mathrm{Fe}$ total & \begin{tabular}{|ll}
$\mathrm{Fe}$ & libre \\
$\mathrm{Fe}$ & total
\end{tabular} & argile & $\begin{array}{l}\text { limons } \\
\text { fins }\end{array}$ & $\begin{array}{c}\text { limons } \\
\text { grossiers }\end{array}$ & $\begin{array}{l}\text { sables } \\
\text { fins }\end{array}$ & $\begin{array}{c}\text { sables } \\
\text { grossiers }\end{array}$ & $\frac{\text { limons } F \text {. }}{\text { argile }}$ \\
\hline (B) & 1,68 & 0,54 & 12 & 36 & 16,5 & 7,7 & 19,7 & 3 \\
\hline II $\mathrm{A}_{2}$ & 3,76 & 0,57 & 39,5 & 26 & 15 & 4,1 & 9 & 0,65 \\
\hline II B/C & 5,64 & 0,66 & 57 & 11 & 5 & 5,4 & 15,2 & 0,19 \\
\hline $\mathrm{II} \mathrm{B/C}$ & 3,90 & 0,53 & 36 & 33 & 12 & 3,5 & 6,4 & 0,97 \\
\hline III C & 3,30 & 0,49 & 12 & 3 & 0 & 3 & 83,4 & \\
\hline
\end{tabular}




\subsection{Essai de détermination}

Seule est concernée dans ce chapitre la partie rubéfiée développée sur ces limons, autrement dit les horizons II $\mathrm{A}_{2}$, II $\mathrm{B}$ et II $\mathrm{B} / \mathrm{C}$. La couleur rouge brique de I'horizon II B, probablement due à des oxydes de fer déshydratés, comme l'hématite, ou peu hydratés, comme la goethite, fait immédiatement penser à une pédogenèse sous climat chaud, à période sèche prolongée. La teneur en fer libre, 2, $14 \%$ pour l'horizon II $\mathrm{A}_{2}$ et $3,76 \%$ pour l'horizon II $\mathrm{B}$, est très élevée comparativement aux sols plus récents du reste du massif, même les plus évolués (sols lessivés : horizon $\mathrm{A}_{2} 0,8 \%$, horizon B 2,2\%. Sols bruns lessivés : horizon $\mathrm{A}_{2} 1,0 \%$, B 1,6\%. Sols bruns sur colluvium : horizon (B) $0,70 \%$. Sols bruns acides ou ocre-podzoliques sur grès : horizon $\mathrm{B} 0,55$ à $0,60 \%$. Horizon $\mathrm{B}_{2}$ des podzols $1,0 \%$ ).

Cette teneur élevée en fer libre permet de supposer que ce sol appartient à la classe des sols à sesquioxydes très individualisés de la classification française. Un certain nombre de caractéristiques permettent de rapprocher ces sols de la sous-classe des sols ferrugineux tropicaux. La teneur en aluminium libre est en effet supérieure à celle des sols bruns lessivés ( $2 \%$ environ) et lessivés ( $3 \%$ environ) de la région, mais inférieure à celle existant dans les sols de la série podzolique, 3 à $6 \%$ pour les sols bruns acides cryptopozoliques et ocre podzoliques, $10 \%$ dans 1'horizon $\mathbf{B}_{2}$ des podzols. Il ne semble donc pas qu'il y ait eu une forte libération d'alumine, ni par conséquent, de ferrallitisation.

- L'épaisseur du profil est faible et ne devait guère dépasser $150 \mathrm{~cm}$. Seuls les sols ferrugineux ont un aussi faible développement.

- Il semble donc que l'on puisse classer ce sol dans le grand groupe des sols ferrugineux tropicaux lessivés. Il est en effet probable que l'enrichissement en argile de l'horizon II B soit dû au lessivage, puisque corrélativement il y a enrichissement en fer libre de cet horizon, par rapport à l'horizon II $\mathrm{A}_{2}$. La présence d'enrobements argileux sur les faces des polyèdres de l'horizon II B est également en faveur de cette hypothèse, que nous retiendrons, bien que quelques anomalies texturales puissent être remarquées : 1'horizon II $\mathrm{A}_{2}$ est aussi riche en argile que l'horizon II $\mathrm{B} / \mathrm{C}$, et d'autre part, la courbe cumulative de l'horizon II B recoupe celle des horizons II $\mathrm{A}_{2}$ et II B/C. Ces anomalies doivent avoir pour origine un brassage par solifluxion, qui a perturbé le limon initial, par incorporation irrégulière d'éléments grossiers.

Quelques autres caractères autoriseraient une analogie avec les sols faiblement ferrallitiques. Dans I'horizon II B le taux de saturation n'est que de $27,6 \%$, alors que généralement pour les sols ferrugineux, il est supérieur à $40 \%$. Le pH est légèrement inférieur à 5 , alors que, habituellement, il oscille aux alentours de 6 pour ces mêmes sols. Le rapport limons fins sur argile est de 0,19 en II B et donc assez semblable à celui des sols ferrallitiques. Mais ce critère est assez discutable.

Nous pouvons également essayer de caractériser ce sol suivant les critères de la classification américaine. La distinction entre alfisols et ultisols se fait essentiellement par le taux de saturation de l'horizon B, qui est inférieur à $35 \%$ dans les ultisols. Le taux de saturation étant de $27,7 \%$ en II B, ce sol serait à classer dans les ultisols. Le taux de sesquioxydes libres, alumine $\left(\mathrm{Al}_{2} \mathrm{O}_{3}\right)$ et fer $\left(\mathrm{Fe}_{2} \mathrm{O}_{3}\right)$ de l'horizon II B est de $6,30 \%(0,93 \%+5,37 \%)$. Par rapport à l'argile $(57 \%)$ ce taux de sesquioxydes libres est de $11,05 \%$. Cet horizon II B n'est donc pas un horizon oxique, le critère de carac- 
térisation étant un taux de sesquioxydes libres par rapport à l'argile supérieur à $12 \%$. Ce sol est cependant proche de la limite entre ultisols et oxisols.

Pour définir les caractéristiques de la pédogenèse, l'étude des types d'argile est importante. Il n'était pas possible ici d'effectuer une telle étude. Nous pouvons cependant approximativement définir le type d'argile par le complexe absorbant. Dans 1'horizon II B le complexe absorbant est de 21,4 m.e. (acétate d'ammonium à pH 7), ce qui correspond à 37 m.e. pour 100 grammes d'argile. L'argile serait donc à dominante d'illite, ce qui est en contradiction avec une pédogenèse de climat chaud. Cependant, la teneur initiale du limon en argile étant de 35 à $40 \%$, la maieure partie de cette argile devait être présente avant la pédogenèse.

\subsection{Conclusion}

Nous pouvons donc estimer que ce sol fossile, selon la classification française, est un sol lessivé désaturé, ferrugineux. D’après la classification américaine, ce sol appartient à l'ordre des ultisols, sols à horizon argillique très fortement désaturés sans horizon oxique. Ces conclusions doivent cependant être prises avec une certaire réserve. En effet, ce sol a été soumis après sa formation à des conditions climatiques différentes : il a été recouvert par une nouvelle couche limoneuse ayant par la suite plus ou moins disparu et ayant été remplacée par un colluvium de faible épaisseur $(60 \mathrm{~cm})$. Cette faible épaisseur ne soustrait pas entièrement ce sol fossile à l'action de la pédogenèse actuelle. II n'est donc pas impossible que certaines caractéristiques originelles aient été modifiées. La plupart du temps, il est d'ailleurs impossible de distinguer des horizons. Il ne reste qu'une zone rubéfiée, correspondant probablement à l'horizon II B. Tous ces sols sont donc pour la plupart tronqués. Souvent aussi ils ont entièrement disparu.

\subsection{Age de ce sol}

Le matériel sur lequel s'est constitué ce sol ancien est un limon manifestement d'origine éolienne. En aucun cas, ce matériel ne peut être considéré comme un faciès limono-argileux des alluvions anciennes, bien que la teneur en cailloux roulés puisse atteindre $30 \%$; en effet, ces limons toujours situés à la partie supérieure des alluvions, reposent directement sans transition sur les éléments grossiers sous-jacents, et ne présentent aucune trace de stratification entrecroisée. La présence des cailloux roulés s'explique facilement par la solifluxion. Il est remarquable de constater que les phénomènes de solifluxion n'ont pas perturbé les horizons du sol fossile. Par conséquent, la pédogenèse est postérieure à la solifluxion. Ce sol fossile, développé sur un matériel éolien soliflué, donc mis en place au cours d'une période froide, est d'âge quaternaire.

Rien ne permet de le rattacher à l'une des 3 périodes interglaciaires. Cependant, on admet que pendant les deux premières périodes interglaciaires régnait un climat plus chaud que le climat actuel, la flore du deuxième interglaciaire indiquant déjà une influence tempérée. Nous avons vu que le climat qui régnait lors de la pédogenèse était très chaud à caractère tropical et ne peut correspondre à celui qui règnait au 
deuxième interglaciaire. Par conséquent ce sol rubéfié pourrait dater des premières phases du quaternaire. Il en serait de même de toutes les formations rubéfiées, développées sur limons, qui ont été très fréquemment rencontrées à la tarière sous des sols plus récents, qui vont maintenant être étudiés.

\section{3. - SOLS LESSIVÉS A PSEUDOGLEY}

Ces sols sont bien représentés dans le massif forestier étudié. Ils se rencontrent exclusivement au sommet de l'ancienne terrasse alluviale, en position horizontale, et sur un placage de limons éoliens qui recouvrent le sommet de cette terrasse.

\subsection{Origines possibles et conséquences de l'existence d'un niveau imperméable}

Tous les sols développés sur limons sont caractérisés par l'existence d'un niveau imperméable, limono-argileux, ocre-rouille, parcouru par un réseau de marbrures blanchâtres décolorées, caractéristiques des horizons de pseudogley et constituant le plancher d'une nappe temporaire. Cette nappe apparait immédiatement après une pluie et disparaît plus ou moins rapidement. Cet horizon imperméable est situé à une profondeur variable, comprise entre 30 et $120 \mathrm{~cm}$.

Lorsqu'il est situé à $30 \mathrm{~cm}$ de profondeur, les caractéristiques du profil sont essentiellement dominées par l'hydromorphie. Que l'horizon imperméable soit dû à l'existence de deux couches géologiquement différentes ou qu'il soit secondaire et résulte d'un processus pédologique, le sol appartient aux pseudogleys à nappe perchée (classe des sols hydromorphes). Par contre, lorsque cet horizon argileux se trouve à 1 mètre de profondeur, les caractères d'hydromorphie ne sont plus que secondaires et, génétiquement, le sol est à ranger dans la classe des sols à mull ; il appartient alors à une sous-classe différente suivant que l'existence de cet horizon argileux est ou n'est pas, la conséquence d'un processus de lessivage. Pour les sols de la Brenne, Mme PERIGAUD estime sans être affirmative que cette hydromorphie s'est superposée à une évolution antérieure, en l'occurence, un lessivage ancien intense. Pour les sols de la forêt de Chaux, PLAISANCE estime également que le lessivage est intervenu dans leur formation, et que ces sols sont au moins en partie d'origine ancienne. A Sainte-Hélène, la cartographie détaillée a montré que sur tout le placage limoneux existaient deux " couches » superposées, l'une limoneuse claire (2,5 Y 7/4), l'autre limono-argileuse plus foncée $(2,5$ Y $7 / 8)$, toujours située sous la précédente. Jamais la couche inférieure n'apparaît seule, et jamais la couche supérieure ne dépasse 1 mètre. Une telle constance fait immédiatement penser à la possibilité d'une laison entre ces deux couches. C'est pour ces raisons, qu'en première approximation, dans les descriptions qui vont suivre, ces couches ont été respectivement appelées horizon $\mathrm{A}_{2}$ et horizon $\mathrm{B}$.

Deux profils situés dans des conditions analogues, sous futaie de hêtres et de chênes en mélange, ont été étudiés d'une manière détaillée afin de déterminer si une telle liaison pédologique existe et, dans cette hypothèse, de préciser la nature de cette liaison. 


\subsection{Etude et comparaison de deux profils}

\subsection{Description}

\section{Profil 1}

Horizon $\mathrm{A}_{11}$ : Mull moder, structure à peine différenciée, agrégats extrêmement fins ; $0-5 \mathrm{~cm}$ transition peu nette avec l'horizon inférieur. Enracinement abondant de la strate herbacée.

Horizon $\mathrm{A}_{12}$ : Horizon nettement influencé par la matière organique. Structure gru$0-30 \mathrm{~cm} \quad$ muleuse irrégulière : grumeaux anguleux de 3 à $5 \mathrm{~mm}$. Très meuble. Enracinement très abondant. 2,5 Y 6/4 (jaune gris).

Horizon $\mathrm{A}_{2}$ : Absence de structure. Canalicules très fins et nombreux, devenant $30-70 \mathrm{~cm}$ encore plus abondants à la partie inférieure de l'horizon. Forte compacité en période de sécheresse. Enracinement abondant. 2,5 Y 7/4 (jaune pâle).

Horizon $\mathrm{A}_{2 \vartheta}$ : de $70 \mathrm{à} 90 \mathrm{~cm}$ : structure fondue ayant une légère tendance à devenir $70-120 \mathrm{~cm} \quad$ polyédrique. 2,5 Y $7 / 4$ avec 10 à $15 \%$ de plages 10 YR $7 / 6$ (jaune) de 90 à $120 \mathrm{~cm}$ : structure polyédrique peu nette.

Juxtaposition de 4 couleurs : matrice 2,5 Y $7 / 4-10$ à $20 \%$ de plages gris clair, 2,5 Y $7 / 2-10$ à $20 \%$ de plages ocre, 10 YR $7 / 6-2$ à $3 \%$ de concrétions rouille, 7,5 YR 7/2. Transition diffuse avec 1'horizon sous-jacent. Enracinement peu abondant.

Horizon $\mathbf{B}_{g}$ : de 120 à $150 \mathrm{~cm}$, traversé par des bandes décolorées verticales blan$120-170 \mathrm{~cm}$ châtres pulvérulentes. Très compact. Structure polyédrique remarquable. Gros polyèdres de 3 à $4 \mathrm{~cm}$ de longueur souvent aplatis. Les faces des polyèdres sont souvent décolorées et pulvérulentes, surtout celles de ceux bordant les bandes blanches. Nombreuses concrétions d'oxydes de fer et de manganèse sur les faces des polyèdres. Présence constante de clay-skins très nets. L'intérieur des polyèdres de couleur 2,5 Y $7 / 8$ (jaune gris) est traversé par un réseau de canalicules dont les parois sont rouille foncé.

de 150 à $170 \mathrm{~cm}$, structure polyédrique ayant tendance à devenir nuciforme. Transition brutale avec l'horizon inférieur.

Horizon II B : prélèvement à la tarière. Structure impossible à déterminer. Trainées $170-200 \mathrm{~cm}$ blanchâtres verticales. 7,5 YR $5 / 8$ (jaune rougeâtre) à la partie supérieure, puis 5 YR $5 / 8$ (rouge jaune) entre $150 \mathrm{et} 200 \mathrm{~cm}$, à nouveau 7,5 YR $5 / 8$.

A partir de $210 \mathrm{~cm}$, nombreux cailloux roulés empêchant le prélèvement.

\section{Profil 2}

Horizon $A_{11}$ : Moder légèrement hydromorphe, très noir. Structure finement gru$1-4 \mathrm{~cm}$ muleuse. Transition nette avec l'horizon inférieur. 
Horizon $\mathrm{A}_{12}$ : Structure grumuleuse : grumeaux de I à $5 \mathrm{~mm}$ en moyenne, parfois 4 - $10 \mathrm{~cm} \quad 1 \mathrm{~cm}$, de forme angulaire. Enracinement dense, 2,5 Y 7/4 (jaune pâle).

Horizon $\mathrm{A}_{2 g}$ : de 20 à $30 \mathrm{~cm}$ : identique à $\mathrm{A}_{2}$, mais 1 à $2 \%$ de taches rouille $10 \mathrm{YR}$ $20-45 \mathrm{~cm} \quad 7 / 6$. Structure fondue.

de 30 à $45 \mathrm{~cm}$ : Structure fondue à légère tendance polyédrique. Enracinement peu abondant. Juxtaposition de 4 couleurs. Matrice 2,5 Y $7 / 4-10$ à $20 \%$ de plages claires, 2,5 Y $2 / 7-20$ à $25 \%$ de plages rouille, 10 YR 7/6, 2 à $3 \%$ de taches rouille foncé 7,5 Y $6 / 6$. Transition nette avec l'horizon sous-jacent.

Horizon $\mathrm{B}_{g}$ : Structure polyédrique à grosses unités de 3 à $5 \mathrm{~cm}$ de longueur, ayant $45-180 \mathrm{~cm}$ tendance à devenir lamellaire vers $80 \mathrm{~cm}$. Clay skins. Nombreuses bandes décolorées verticales, surtout à la partie supérieure. Couleur dominante 10 YR 6/6 (jaune), quelques rares taches rouille 7,5 Y 6/8. Abondantes concrétions d'oxydes de fer et de manganèse.

De 90 à $180 \mathrm{~cm}$ : prélèvement à la tarière. Couleur identique. Absence presque totale de bandes décolorées verticales.

Horizon B/C : Teinte générale 2,5 Y 7/6 (jaune). $30 \%$ de plages 10 YR $6 / 6$. $180-200 \mathrm{~cm}$

Horizon II B: Transition très brutale. Couleur générale 7,5 YR 6/8 (jaune rouge) $200-260 \mathrm{~cm}$ traces importantes d'hydromorphie dans la zone de transition.

Morphologiquement ces 2 profils distants de $1800 \mathrm{~m}$ diffèrent essentiellement par la profondeur d'apparition de l'horizon limono-argileux $(\mathrm{Bg}), 120 \mathrm{~cm}$ pour le profil $\mathrm{n}^{\circ} 1$ et $45 \mathrm{~cm}$ pour le profil $\mathrm{n}^{\circ} 2$. Deux autres différences peuvent être remarquées : l'horizon limono-argileux du profil 2 présente un aspect plus bigarré que celui du profil 1. D'autre part, I'humus dans un cas est un moder légèrement hydromorphe et dans l'autre cas, un mull-moder. Sous I'horizon limono-argileux, apparait brutalement dans les 2 cas, respectivement à 170 et $210 \mathrm{~cm}$, un horizon rubéfié jaune rouge (II B). L'étude précédente des formations rubéfiées a montré que nous étions en présence des restes d'un ancien sol développé en climat chaud. Dans ce chapitre, nous ne tiendrons compte que de la partie située au-dessus du limon rubéfié.

\subsection{Répartition du fer et de Pargile}

Des prélèvements ont été faits tous les $10 \mathrm{~cm}$ et, sur chaque échantillon, le dosage du fer libre et une analyse granulométrique ont été effectués, ce qui a permis de dresser les graphiques de répartition du fer libre et de l'argile en fonction de la profondeur.

\section{Profil I}

Les deux courbes du fer et de l'argile sont concordantes et permettent de penser à l'intervention du lessivage (fig. 1 et 3 ). On peut en effet, distinguer un horizon $\mathrm{A}_{2}$ de $100 \mathrm{~cm}$ environ, et un horizon B beaucoup moins épais de $60 \mathrm{~cm}$. Cette disproportion entre les 2 horizons s'explique aisément si l'on admet que l'accumulation du fer et de 
l'argile a dû s'effectuer sur une plus grande épaisseur. Du fait de l'hétérogénéité de la roche-mère, il est impossible de le mettre en évidence. Nous sommes en présence d'un profil incomplet par sa partie inférieure, qui se confond avec I'horizon II B du sol fossile. Si nous calculons les indices d'entraînement du fer et de l'argile en prenant les valeurs maxima et minima, nous obtenons respectivement 2,37 et 2,1 , ce qui permet de classer ce sol dans la classe des sols lessivés, en admettant que la roche-mère soit bien homogène.

L'examen des courbes cumulatives des prélèvements effectués à $35 \mathrm{~cm}\left(\mathrm{~A}_{2}\right)$, $135 \mathrm{~cm}\left(\mathrm{~B}_{g}\right)$ et $185 \mathrm{~cm}$ (limon ancien) permet de conclure en faveur de l'homogénéité du matériel initial jusqu'à $170 \mathrm{~cm}$ (fig. 5). Les courbes des horizons $\mathrm{A}_{2}$ et $\mathbf{B}_{y}$ sont constamment situées l'une au-dessus de l'autre, leur décalage correspondant à la quantité d'argile ayant migré par lessivage. La courbe cumulative du limon ancien recoupe les courbes des 2 horizons $\mathrm{A}_{2}$ et $\mathrm{B}_{g}$, ce qui semble indiquer un matériel différent (fig. 5). Une nouvelle confirmation de cette interprétation peut être obtenue en utilisant les 3 courbes cumulatives précédentes. On peut en effet construire 3 nouvelles courbes cumulatives en ne tenant compte que des fractions comprises entre 2 et 2000 microns. De cette façon, on élimine l'argile, qui est la seule fraction pouvant migrer par lessivage; les courbes ainsi obtenues sont caractéristiques de la roche-mère et indépendantes de tout phénomène pédologique (fig. 7). Par ce procédé, la superposition des courbes cumulatives des horizons $\mathrm{A}_{2}$ et $\mathrm{B}_{g}$ est presque parfaite. Par contre, la courbe de I'horizon II B du limon rubéfié ne se superpose pas aux deux autres courbes. II semble donc bien que les différences granulométriques observées entre les horizons $\mathrm{A}_{2}$ et $\mathrm{B}_{g}$ soient uniquement dues au lessivage.

\section{Profil 2}

Ici, le limon ancien apparaît à une profondeur plus importante, ce qui permet d'effectuer un bilan sur $210 \mathrm{~cm}$. Comme le précédent profil, on peut distinguer deux horizons : un horizon $\mathrm{A}_{2}$ de 35 à $40 \mathrm{~cm}$ et un horizon $\mathrm{B}_{g}$ de $130 \mathrm{à} 140 \mathrm{~cm}$. Comme pour le profil 1 , nous observons une disproportion entre les horizons lessivés et d'accumulation, mais cette fois au bénéfice de l'horizon $\mathrm{B}_{g}$ (fig. 2 et 4). En effet, le limon ancien est suffisamment profond pour que l'horizon $\mathbf{B}_{g}$ puisse être mis en évidence sur toute son épaisseur. Par contre, le très faible développement de l'horizon $A_{2}$ ne peut s'expliquer que par sa disparition partielle du fait de l'érosion.

Enfin, si l'on compare les courbes du fer et de l'argile, il n'y a correspondance que dans leur partie supérieure. A partir de $1 \mathrm{~m}$ et jusqu'à $2,30 \mathrm{~m}$, le taux d'argile est constant en dépit de petites variations.

Le taux d'argile de l'horizon $\mathrm{B}_{\vartheta}$ proprement dit est identique à celui de l'horizon B/C. Il n'y a pas de " ventre » d'accumulation. Il est donc impossible de dire d'après la courbe de l'argile si la différence entre $\mathrm{A}_{2}$ et $\mathrm{B}_{g}$ est due au lessivage. Par contre, la courbe de fer libre montre ce " ventre $»$ d'accumulation et, par conséquent, une différence entre $\mathrm{B}_{g}$ et $\mathrm{B} / \mathrm{C}$.

On peut attribuer l'absence de différence entre le taux d'argile des horizons $\mathbf{B}_{y}$ et $\mathrm{B} / \mathrm{C}$, soit à une accumulation diffuse, sur une plus grande épaisseur que dans le cas du fer, soit à une légère hétérogénéité en profondeur du matériel initial. On peut également penser à une destruction d'argile en $\mathbf{B}_{g}$, mais le taux d'alumine libre étant cons- 
TABLEAU 2

Caractéristiques analytiques du profil I

\begin{tabular}{|c|c|c|c|c|c|c|c|c|c|c|c|c|c|c|c|}
\hline & \multirow{2}{*}{$\begin{array}{l}\mathrm{pH} \\
\text { eau }\end{array}$} & \multirow{2}{*}{$\mathrm{M}, \mathrm{O}$} & \multirow{2}{*}{$\mathrm{C} / \mathrm{N}$} & \multicolumn{4}{|c|}{$\mathrm{me} / 100 \mathrm{~g}$} & \multirow{2}{*}{$\mathrm{S} / \mathrm{T}$} & \multirow{2}{*}{$\begin{array}{l}\text { Al libre } \\
\text { (Tamm) } \\
\% \text { oo }\end{array}$} & \multirow{2}{*}{$\begin{array}{c}\text { Fe libre } \\
\text { (DEB) } \\
\%\end{array}$} & \multirow{2}{*}{ Argile } & \multirow{2}{*}{$\begin{array}{l}\text { Limons } \\
\text { fins }\end{array}$} & \multirow{2}{*}{$\begin{array}{l}\text { Limons } \\
\text { grossiers }\end{array}$} & \multirow{2}{*}{$\begin{array}{c}\text { Sables } \\
\text { fins }\end{array}$} & \multirow{2}{*}{$\begin{array}{l}\text { Sables } \\
\text { grossiers }\end{array}$} \\
\hline & & & & K & $\mathrm{Ca}$ & $\mathrm{Mg}$ & $\mathrm{T}$ & & & & & & & & \\
\hline$A_{1}$ & 4,4 & 19,9 & 17,8 & 1,21 & 4,20 & 2,41 & 22 & 35 & & & & & & & \\
\hline $\mathrm{A}_{2}$ & 5,1 & 0,7 & & 0,11 & 0,73 & 0,46 & 4 & 32,5 & 2,7 & 0,78 & 16 & 49 & 23 & 6,5 & 7,1 \\
\hline $\mathbf{B}_{g}$ & 5,2 & 0,3 & & 0,19 & 1,30 & 1,30 & 9 & 30 & 3,0 & 1,90 & 33,5 & 35 & 17 & 4,7 & 4,7 \\
\hline
\end{tabular}

TABLEAU 3

Caractéristiques analytiques du profil II

\begin{tabular}{|c|c|c|c|c|c|c|c|c|c|c|c|c|c|c|c|}
\hline & \multirow{2}{*}{$\begin{array}{l}\mathrm{pH} \\
\text { eau }\end{array}$} & \multirow{2}{*}{$\underset{\%}{\text { M.O }}$} & \multirow{2}{*}{$\mathrm{C} / \mathrm{N}$} & \multicolumn{4}{|c|}{$\mathrm{me} / 100 \mathrm{~g}$} & \multirow{2}{*}{$\mathrm{S} / \mathrm{T}$} & \multirow{2}{*}{$\begin{array}{c}\text { Al libre } \\
\text { (Tamm) } \\
\% \text { oo }\end{array}$} & \multirow{2}{*}{$\begin{array}{c}\text { Fe libre } \\
\text { (DEB) } \\
\%\end{array}$} & \multirow{2}{*}{ Argile } & \multirow{2}{*}{$\begin{array}{l}\text { Limons } \\
\text { fins }\end{array}$} & \multirow{2}{*}{$\begin{array}{l}\text { Limons } \\
\text { grossiers }\end{array}$} & \multirow{2}{*}{$\begin{array}{l}\text { Sables } \\
\text { fins }\end{array}$} & \multirow{2}{*}{$\begin{array}{l}\text { Sables } \\
\text { grossiers }\end{array}$} \\
\hline & & & & K & $\mathrm{Ca}$ & $\mathrm{Mg}$ & $\mathrm{T}$ & & & & & & & & \\
\hline$A_{1}$ & 3,8 & 29,9 & 22,3 & 1,31 & 1,55 & 0,83 & 26 & 14,2 & & 0,83 & & & & & \\
\hline $\mathrm{A}_{2 g}$ & 4,7 & 0,7 & & 0,28 & 0,25 & 0,11 & 5,5 & 11,6 & 2,8 & 0,74 & 12,5 & 52 & 22 & 5,2 & 5,8 \\
\hline $\mathrm{B}_{g}$ & 5,05 & & & 0,22 & 0,44 & 1,46 & 10 & 21 & 3,2 & 1,82 & 33 & 39 & 15,5 & 2,9 & 3,1 \\
\hline $\mathrm{B}_{g}$ & 5,1 & & & 0,19 & 1,53 & 2,16 & 11 & 32,8 & 3,1 & 2,15 & & & & & \\
\hline $\mathrm{B} / \mathrm{C}$ & 5,35 & & & 0,21 & 2,32 & 2,33 & 11,5 & 42,0 & 3,2 & 1,34 & & & & & \\
\hline
\end{tabular}




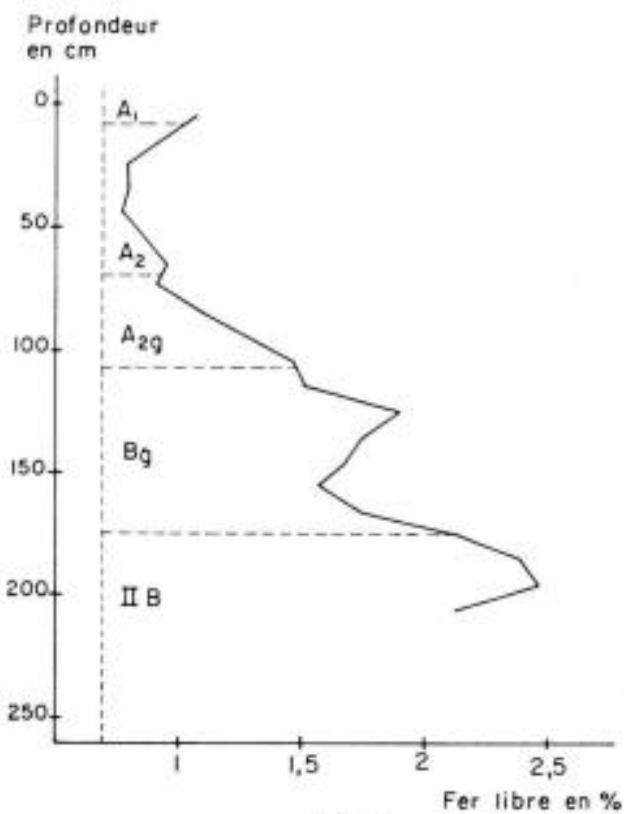

Fig. 1

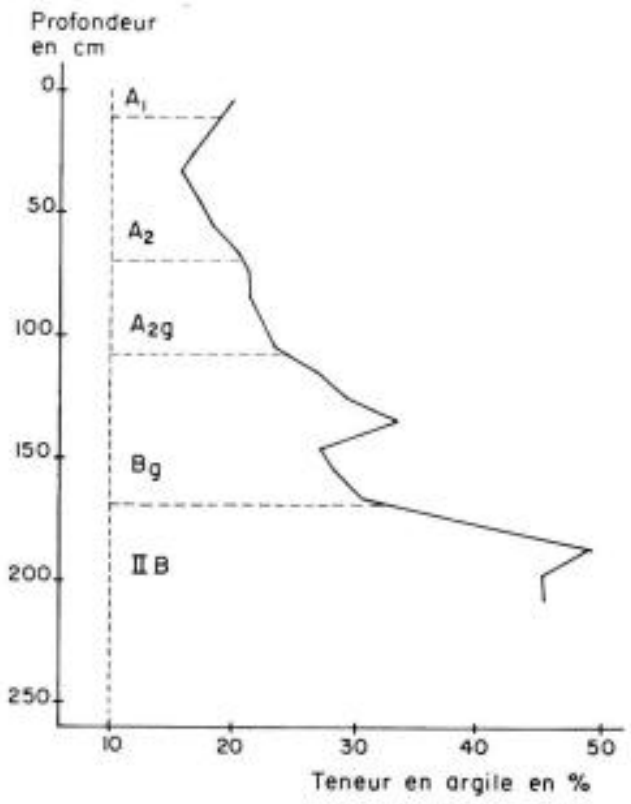

Fig. 3

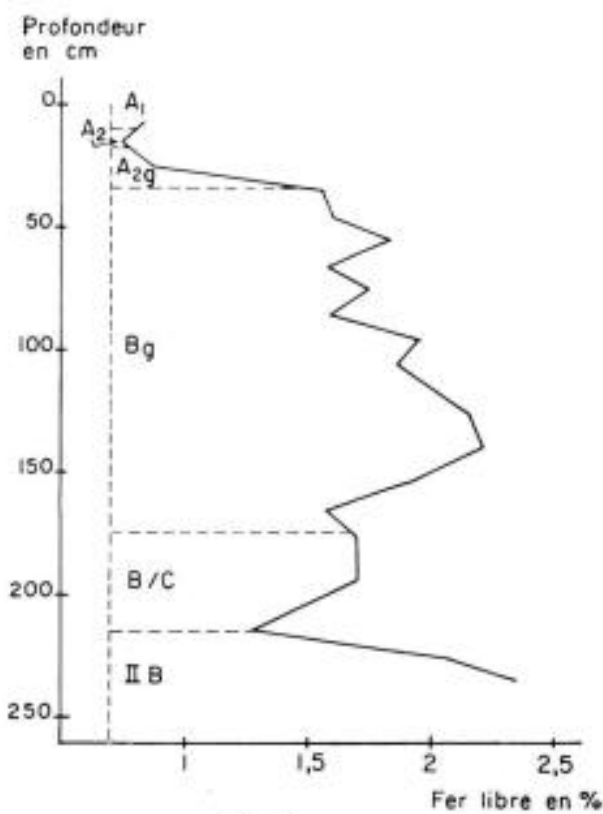

Fig. 2

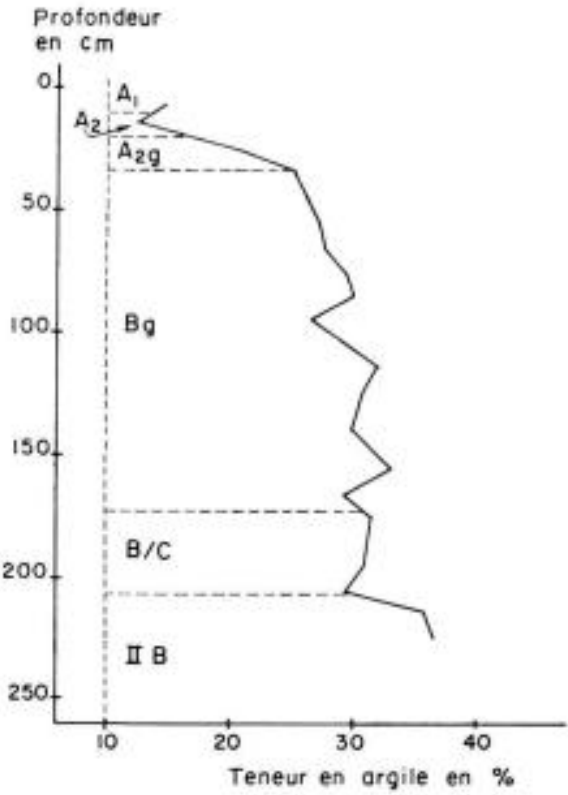

Fig. 4

FIG. 1. - Profil 1 : teneur en fer libre en fonction de la profondeur

FIG. 2. - Profil II : teneur en fer libre en fonction de la profondeur

Fig. 3. - Profil I : teneur en argile en fonction de la profondeur

Fig. 4. - Profil II : teneur en argile en fonction de la profondeur 
Fig. 5
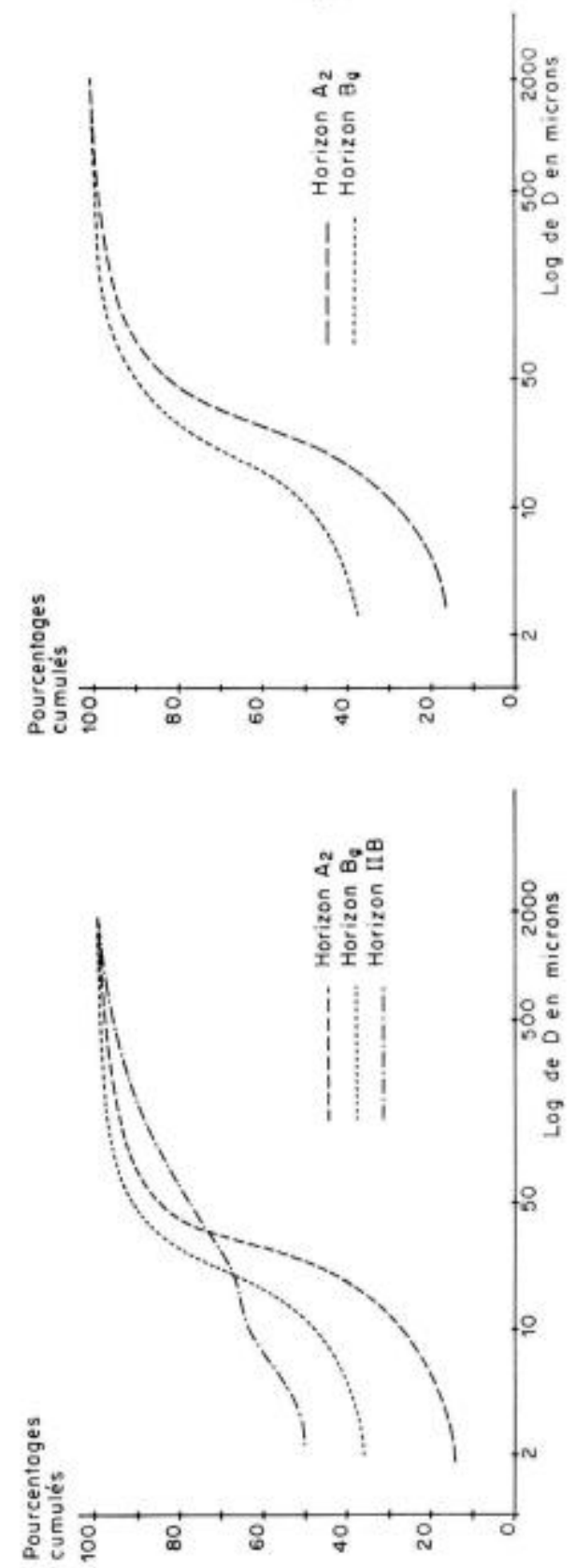

Fig. 7
Fig. 6
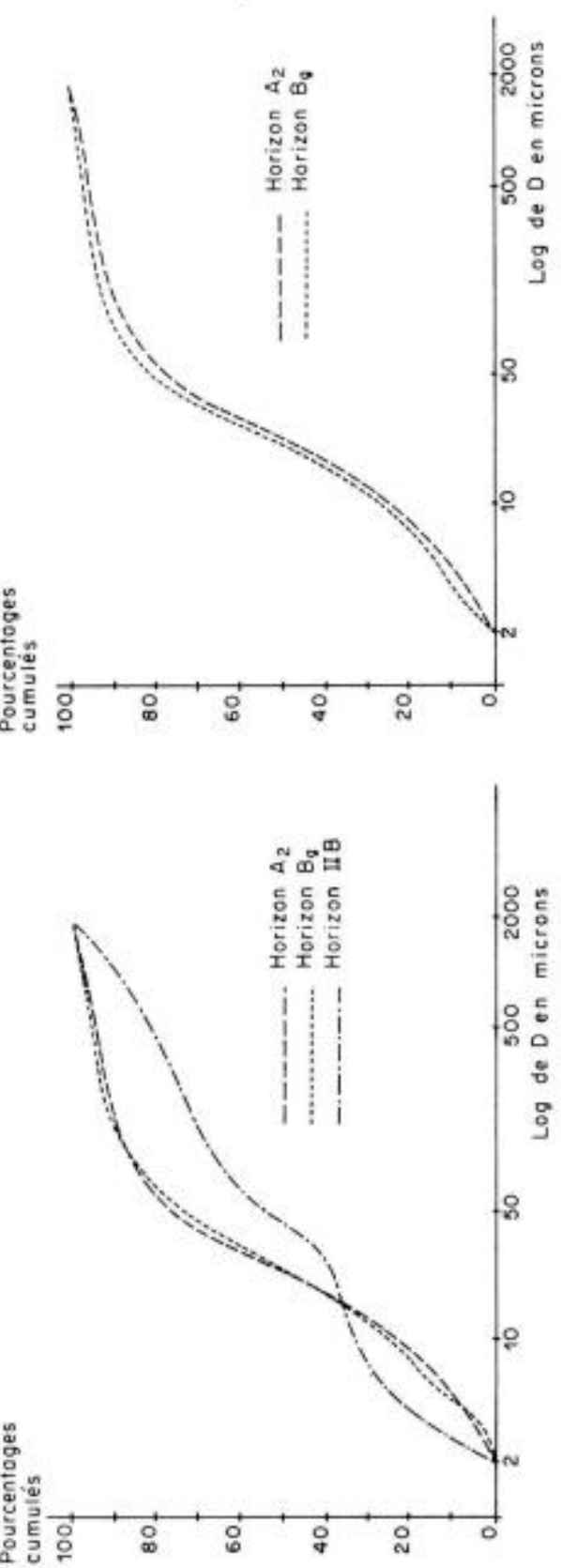

Fig. 8

FIG. 5. - Profil 1 : courbes cumulatives de 0 à $2000 \mu$ des horizons $A_{2}, B_{g}$, et II B

Fic. 6. - Profil II : courbes cumulatives de 0 à $2000 \mu$ des horizons $A_{2 g}$ et $B_{g}$

Fig. 7. - Profil $I$ : courbes cumulatives de 2 à $2000^{2}$ des horizons $A_{2}, B_{g}$ et IIB par élimination de l'argile

Fic. 8. - Profil : courbes cumulatives de 2 à $2000 \mu$ des horizons $A_{2 g}$ et $B_{g}$, par élimination de Targile 


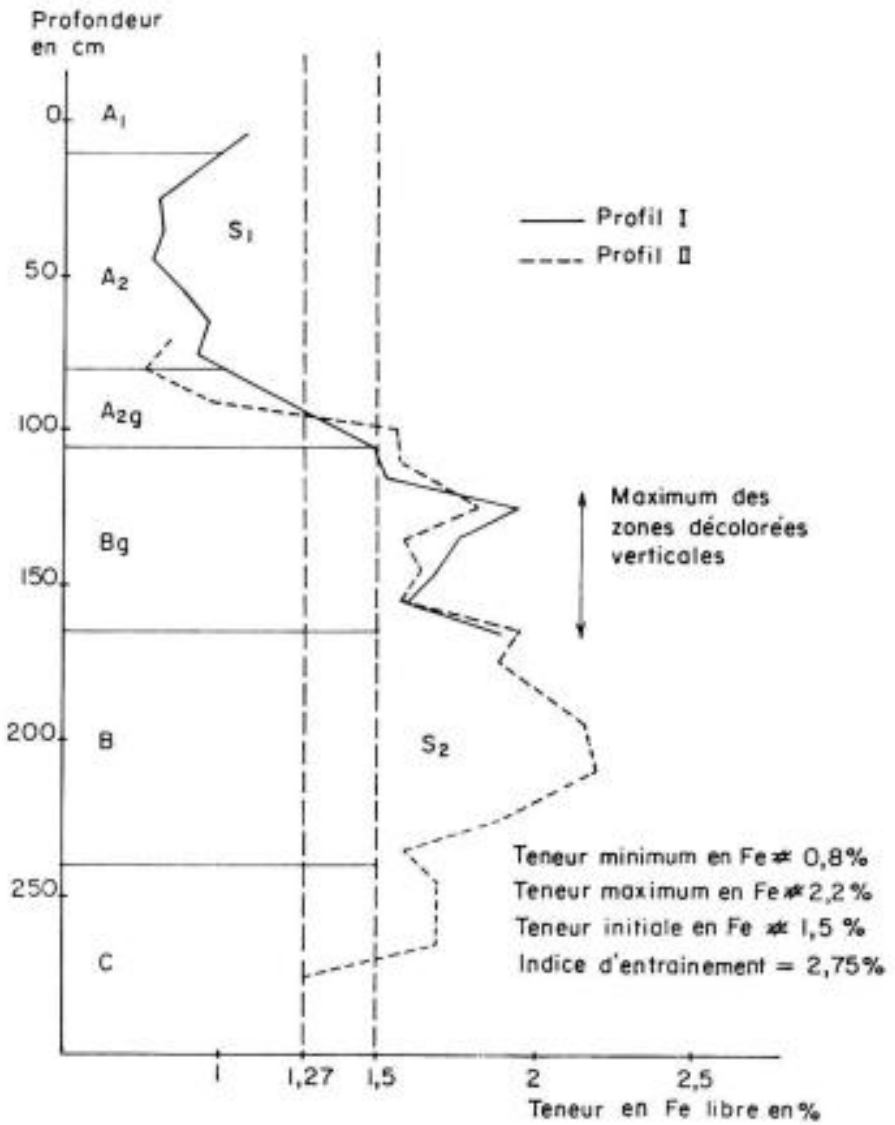

Fig. 9. - Superposition des graphiques de la teneur en fer libre en fonction de la profondeur des profils I et II 
tant dans tout le profil, il ne semble pas que ce fait ait pu jouer ici. L'existence de ce " ventre » d'accumulation du fer libre permet cependant de penser que seul le lessivage intervient dans la différenciation des deux horizons $\mathrm{A}_{2}$ et $\mathrm{B}_{g}$. Les courbes cumulatives correspondant à des prélèvements effectués à $15 \mathrm{~cm}\left(\mathrm{~A}_{2}\right)$ et $140 \mathrm{~cm}\left(\mathrm{~B}_{\mathrm{g}}\right)$, le confirment. Nous obtenons comme pour le précédent profil deux courbes superposées qui ne se chevauchent jamais (fig. 6). De même, comme pour le profil $n^{\circ} 1$, les courbes cumulatives des horizons $\mathrm{A}_{2}$ et $\mathrm{B}_{g}$, effectuées de 2 à 2000 microns, donc sans tenir compte de l'argile, se superposent et permettent de conclure dans le même sens (fig. 8).

\subsection{Superposition des Profils I et II}

L'examen des courbes cumulatives des deux horizons $\mathrm{A}_{2}$ et $\mathrm{B}_{g}$ des profils I et II montre qu'elles sont presque superposables deux à deux. Le matériel initial était donc identique au départ, et le lessivage a été égal en intensité dans les deux cas. Cependant, le résultat a été la différenciation de 2 profils dissemblables par l'absence de la partie inférieure en I, due à l'insuffisance d'épaisseur du limon, et par l'absence en II de la partie supérieure, due à l'érosion. Pour obtenir un profil complet, on peut donc essayer de superposer les profils I et II. L'utilisation des courbes de lessivage de l'argile ne donne guère de résultats, probablement du fait de la microhétérogénéité du limon initial. Par contre, on peut supposer que la teneur en fer libre était initialement indépendante de la microhétérogénéité de la texture et constante sur toute l'épaisseur du limon. La superposition des courbes du fer libre a été effectuée en faisant coïncider les parties correspondant aux horizons $\mathbf{B}_{g}$, et qui sont seules communes (fig. 9). On obtient ainsi une courbe complète presque parfaitement symétrique par rapport à un axe représentant la teneur initiale en fer libre. On peut estimer cette teneur à $1,5 \%$ en faisant la moyenne des teneurs maxima et minima observées en $\mathrm{A}_{2}$ et $\mathrm{B}$. Les deux surfaces $S_{1}$ et $S_{2}$ sont ainsi égales. On peut estimer aussi que la teneur initiale en fer libre était proche de la teneur minimum observée en B/C, c'est-à-dire 1,27\%.

Cette courbe théorique du fer permet de concevoir un profil complet qui se serait ainsi développé sur $2,70 \mathrm{~m}$ d'épaisseur, ce qui semble considérable. L'horizon $\mathrm{A}_{2}$ aurait environ $1 \mathrm{~m}$ et l'horizon $\mathrm{B}, 1,30 \mathrm{~m}$ à $1,40 \mathrm{~m}$ d'épaisseur. L'indice d'entraînement du fer serait de 2,75 et celui de l'argile de 2,1 à 2,3 .

\subsection{Autres critères de lessivage}

\section{- alumine libre}

Un travail récent de Duchaufour et Souchier a montré que le critère de l'alumine libre pouvait servir à caractériser l'évolution physicochimique des profils. Ces auteurs admettent les teneurs moyennes suivantes, en alumine libre : moins de $2 \%$ dans les sols bruns lessivés, 2 à $6 \%$, dans les sols bruns acides et les sols ocre-podzoliques, plus de $10 \%$ dans l'horizon B des podzols. Pour les deux profils étudiés, nous obtenons une teneur de $3 \%$ en alumine libre, ce qui place exactement ces sols entre les sols bruns lessivés caractérisés par une faible altération des silicates et un entraînement modéré de l'argile et du fer, et les sols ocre-podzoliques ou bruns acides carac- 
térisés par une forte altération, véritable podzolisation chimique sans entrainement. Dans les deux profils I et II, la teneur en alumine libre étant constante dans tous les horizons, l'entraînement de cet élément est nul et la destruction d'argile observée dans certains cas par des auteurs étrangers, inexistante.

\section{- enrobements}

Dans les horizons B, les enrobements argileux sont très visibles. Les faces des polyèdres ont été prélevées par grattage et les pellicules ainsi obtenues soumises à l'analyse granulométrique. A l'endroit du prélèvement, le taux moyen d'argile était de $30 \%$ environ, celui des pellicules obtenues par grattage de $48 \%$. La différence, bien que significative, est faible car les enrobements semblaient uniquement constitués d'argile.

Malgré la grosseur des unités structurales, à chaque grattage, on devait enlever non seulement les enrobements mais aussi une partie du limon non enrichi.

\subsection{Modifications dues à l'hydromorphie}

Il semble donc que la différenciation d'un horizon limoneux reposant sur un horizon limono-argileux soit la conséquence d'un lessivage particulièrement important. Actuellement, le caractère de sol lessivé est masqué par l'hydromorphie qui a provoqué des modifications importantes dans le profil. La partie supérieure de l'horizon $\mathrm{B}$ présente un réseau de bandes verticales blanches, diminuant de densité avec la profondeur, pour disparaître à peu près complètement en $\mathrm{B} / \mathrm{C}$. Le fer a, à peu près, entièrement disparu de ces bandes, par migration latérale. L'indice d'entraînement local est de 12 (partie blanche : $0,30 \%$; partie rouille de bordure : $3,40 \%$; chiffres empruntés à $\mathrm{Ph}$. Duchaufour : ( Tableaux analytiques des sols $)$ ). Ces caractères d'hydromorphie, décrits par PLAISANCE sous le nom de marmorisation contrastée, caractérisent l'horizon $\mathrm{B}_{g}$.

Si des migrations latérales du fer se produisent au niveau des bandes verticales, on peut penser que l'hydromorphie provoque également des migrations verticales secondaires surimposées aux migrations antérieures dues au lessivage. Examinons en effet la courbe théorique de lessivage du fer obtenue par superposition des courbes des profil I et II (fig. 9). On constate, aussi bien pour les profils I et II, au niveau maximum des bandes décolorées, une diminution de la teneur en fer libre sur $40 \mathrm{~cm}$ environ. Cette diminution peut s'expliquer de différentes manières. On peut supposer qu'il y a eu, soit migration ascendante, soit migration descendante du fer. L'allure de la courbe ne permet pas de trancher. D'autre part, au niveau du plancher imperméable, les oxydes de fer et de manganèse précipitent mutuellement et constituent de petites concrétions. Le fer immobilisé dans ces concrétions n'est probablement pas extrait lors de l'extraction par la méthode DEB. On peut donc également estimer que cette diminution de la teneur en fer libre au niveau du plancher de la nappe peut être due à la formation de concrétions d'oxydes de fer et de manganèse.

Enfin I'hydromorphie provoque dans 1'horizon $\mathrm{A}_{2}$, qui devient alors $\mathrm{A}_{2 g}$, des migrations et des reprécipitations de fer très localisées. En $\mathrm{A}_{2 g}$, on observe en effet une couleur générale beige pâle, identique à celle de l'horizon $\mathrm{A}_{2}$ initial et parse- 
mée de plages gris clair (10 à $20 \%$ ), d'où le fer a en partie disparu, de plages jaune rouille $(20$ à $25 \%)$, et de taches franchement rouille ( 2 à $5 \%$ ), oủ le fer a migré. Par opposition à la marmorisation contrastée de l'horizon B, PLAISANCE a appelé ce phénomène " marmorisation floue $»$.

\subsection{Genèse des sols à pseudogley sur limons}

Ces différentes conclusions nous permettent de donner un schéma de la genèse des sols à pseudogley de ce massif.

Dans un premier temps le lessivage provoque la formation d'un horizon d'accumulation qui devient progressivement imperméable. En période de pluie, au-dessus de cet horizon imperméable, donc à la partie inférieure de l'horizon $\mathrm{A}_{2}$, se forme une nappe perchée temporaire. Dans cet horizon très limoneux peu structuré et bien homogène, le fer sous l'action de l'eau chargée de matières organiques réductrices, migre localement et reprécipite en période de sécheresse sans direction préférentielle. Cette marmorisation floue caractérise la zone d'oscillation de la nappe temporaire (horizon $\mathrm{A}_{2 g}$ ). En $\mathrm{B}$ l'eau pénètre lentement et préférentiellement entre les unités structurales, et par les fentes de dessication. Ces zones de passages préférentielles perdent progressivement leur fer libre, d'où l'aspect de cet horizon $\mathbf{B}$ qui devient un horizon $\mathrm{B}_{g}$ (marmorisation contrastée). Ainsi, pour les sols, de SainteHélène, on peut admettre que la marmorisation de l'horizon $\mathrm{B}$ et celle de l'horizon $\mathrm{A}_{2}$ sont les conséquences d'un seul et même phénomène, l'imperméabilisation de l'horizon B par lessivage. Ces deux types de marmorisation ne sont donc pas d'âge différent.

On peut d'autre part estimer que l'intensité de la marmorisation est sous la dépendance de deux facteurs: l'érosion et la végétation. La cartographie a montré que sur tout le placage limoneux, I'horizon B est toujours semblable, avec une teneur en argile et en fer constante. L'horizon lessivé par contre est très variable, son épaisseur pouvant être comprise entre $30 \mathrm{et} 120 \mathrm{~cm}$. Cette variation ne peut être due, semble-t-il, qu'à l'action de l'érosion, qui aurait ainsi tronqué la plupart de ces sols. Cette érosion a eu pour conséquence de rapprocher le niveau imperméable de la surface et d'augmenter la durée et la hauteur de la nappe temporaire ; le volume de sol où se répartit l'eau de pluie a en effet été considérablement réduit après érosion. D'autre part, la végétation ligneuse intervient en utilisant une grande quantité d'eau. Son élimination par une coupe, par exemple, provoquera également une augmentation de la durée et de la hauteur de la nappe temporaire. L'intensité de I'hydromorphie parait donc en relation étroite avec les changements actuels de végétation, mais aussi et surtout avec l'intensité de l'érosion, érosion qui est en rapport avec des phénomènes anciens. De même la fertilité actuelle de ces sols, qui est d'autant plus faible que l'hydromorphie est plus importante et proche de la surface, dépend de cette évolution ancienne.

\subsection{Difficultés d'appellation}

Tous ces sols ont subi quel que soit leur aspect actuel, une évolution première identique. Secondairement, ils ont tous acquis des caractères d'hydromorphie dont 
l'intensité varie avec le degré d'érosion. Les sols très érodés sont hydromorphes dès la surface :

1'horizon $A_{1}$ est en général un moder hydromophe, l'horizon $A_{2 g}$ commence immédiatement sous l'horizon $\mathrm{A}_{1}$. Le profil est $\mathrm{A}_{1} \mathrm{~A}_{2 g} \mathrm{~B}_{g}$. Lorsque l'on passe à des sols de moins en moins érodés, les caractères d'hydromorphie apparaissent de plus en plus profondément, l'horizon $\mathrm{A}_{2 g}$ étant séparé de l'horizon $\mathrm{A}_{1}$ par l'horizon $\mathrm{A}_{2}$ non modifié. Nous passons donc progressivement d'un sol appartenant à la classe des sols hydromorphes, et au groupe des pseudogleys à nappe perchée, à un sol appartenant à la classe des sols à mull et au groupe des sols lessivés. La limite entre les 2 classes ne peut être qu'arbitraire. Il a été décidé d'utiliser comme critère l'apparition de l'horizon $\mathrm{B}_{\mathrm{g}}$ à moins de $40 \mathrm{~cm}$, ce qui correspond à peu près à l'existence de phénomènes de réduction directement sous l'horizon $\mathrm{A}_{1}$. On aboutit à mettre deux sols ne présentant que de faibles différences, ayant subi une pédogenèse absolument identique, et ne différant que par une érosion un peu plus intense, dans deux classes différentes. Il est néanmoins certain, qu'à partir d'un certain degré, ce sont les caractères d'hydromorphie qui dominent et qui bouleversent, non seulement les propriétés physiques, mais également les propriétés chimiques. Il est intéressant, à ce sujet, de noter la dégradation du complexe absorbant de l'horizon B avec l'accentuation de l'hydromorphie. Initialement, en $\mathrm{B}$ le taux de saturation était de 38 à $40 \%$. II passe à $30 \%$ quand l'horizon de pseudogley est peu marqué puis à $20 \%$ environ quand l'hydromorphie se rapproche de la surface. Enfin, dans le cas du pseudogley véritable, ce taux de saturation est passé à $12 \%$ en $\mathbf{B}_{g}$.

\subsection{Age de ces sols}

Il est particulièrement difficile de se prononcer, et nous ne pouvons avancer que des hypothèses très prudentes. Nous avons pu déterminer que les sols à hydromorphie temporaire de ce massif, étaient des sols lessivés.

Ce sont des sols très évolués dont la formation a dû s'étendre sur une très longue période. On ne peut pas en effet expliquer autrement l'entraînement du fer à plus de $2 \mathrm{~m}$ de profondeur. De plus, bien qu'étant en station horizontale, les $4 / 5$ de ces sols sont très fortement érodés. Dans certains cas, nous pouvons supposer que cette érosion a provoqué la disparition de 70 à $80 \mathrm{~cm}$ de sol. Il est difficile de concevoir une érosion aussi importante lors de la période forestière actuelle. Cette période forestière a débuté dès la fin de la dernière glaciation. II faudrait donc admettre que l'érosion de ces sols lessivés s'est produite juste à la fin du Würm ou pendant le Würm. La formation de ces sols auraient alors eu lieu pendant le dernier interglaciaire Riss-Würm.

\section{4. - RELATIONS POSSIBLES ENTRE LIMONS RUBÉFIÉS ET LIMONS LESSIVÉS}

Il existe très souvent, dans la zone du complexe alluvions-limons, une zone limoneuse rubéfiée profonde correspondant, comme nous l'avons vu dans un chapitre précédent, à des sols formés sous climat chaud. Ces sols sont surmontés par un limon non rubéfié sur lequel se sont développés des sols lessivés à pseudogley. Parfois comme pour 
le profil no 1 étudié précédemment, il y a juxtaposition et confusion partielle des horizons rubéfiés et des horizons d'accumulation des sols lessivés. Il est possible à ce sujet d'émettre deux hypothèses. Nous avons admis l'existence de deux couches superposées, distinctes géologiquement; la couche mise en place le plus anciennement aurait été seule rubéfiée, la seconde aurait été uniquement lessivée, sans être soumise à un processus quelconque de rubéfaction. Il est possible d'interpréter ces faits d'une manière légèrement différente. Nous pouvons en effet supposer qu'il n'existe qu'un seul placage limoneux, qui aurait été entièrement rubéfié sur une très grande épaisseur. Ultérieurement au cours d'une phase de dérubéfaction ou de brunification, la partie supérieure de ce placage limoneux aurait été lessivée, tandis que les oxydes de fer seraient repassés à l'état hydraté. De tels phénomènes ont été observés en Sologne, oủ les sols rubéfiés se sont formés sur des dépôts d'âge Burdigalien. Il existe partout en surface une couche deferrifiée et lessivée qui est géologiquement identique à la couche inférieure rubéfiée sous-jacente. Nous avons, pour le massif étudié, admis la première hypothèse, c'est-à-dire discontinuité géologique et rubéfaction uniquement de la couche limoneuse la plus ancienne. La discontinuité géologique semble être très probable, comme le montrent les courbes cumulatives de texture (fig. 5 et 7 ).

D'autre part, dans une carrière, une couche de limons non rubéfiés repose directement sur le grès bigarré sans l'intermédiaire d'une couche rubéfiée. Ces deux faits permettent donc d'estimer, comme nous l'avons admis, que le dépôt éolien s'est effectué en deux temps.

II est possible cependant, même en admettant cette discontinuité géologique, de supposer que l'ensemble des deux couches a été rubéfié, et que la dérubéfaction, si elle a existé, n'a interessé que la couche supérieure. Pour notre part, nous n'avons pas non plus retenu cette hypothèse. L'examen de la zone de contact, bien qu'elle soit perturbée par des phénomènes de cryoturbation, montre que le passage entre les deux formations est toujours brutal, ce qui semble en contradiction avec l'hypothèse d'une dérubéfaction qui laisserait plutôt supposer une transition progressive.

Nous avons également effectué une étude sommaire de l'intensité de l'altération dans la couche rubéfiée profonde et dans la couche lessivée supérieure. Il est possible d'avoir une idée de l'intensité de l'altération en effectuant les rapports fer libre sur fer total, ou fer total sur fer libre. Plus le rapport fer total sur fer libre est élevé, plus l'altération est faible. Deux profils ont été étudiés: un sol lessivé à pseudogley (profil 1), et le sol rubéfié de la carrière de St-Gorgon. Les horizons $\mathrm{A}_{2}$ et $\mathbf{B}_{g}$ du sol lessivé ont un rapport supérieur à 2. La moyenne du rapport fer total sur fer libre pour l'ensemble de ce sol est de 2,3. Les différents horizons rubéfiés ont tous un rapport fer total sur fer libre inférieur à 2, la moyenne de l'ensemble des horisons étudiés étant de 1,75. Il semble donc bien que l'intensité de l'altération soit plus importante dans les horizons rubéfiés, que dans les horizons qui ne le sont pas, ce qui apporte un argument important à l'hypothèse que nous avons formulée. Pour ces différentes raisons, nous avons admis l'hypothèse suivante :

a) mise en place d'une première couche limoneuse d'origine éolienne,

b) forte altération et rubéfaction de cette couche sous I'action d'un climat à caractère tropical,

c) dépôt d'une seconde couche limoneuse, 

faction.

d) lessivage ancien et intense de cette couche supérieure sans processus de rubé-

\section{5. - SOLS BRUNS LESSIVÉS ET SOLS BRUNS ACIDES}

Il existe des sols bruns lessivés sur limons remaniés et sur grès, ainsi que des sols bruns acides, où l'entrainement du fer et de l'argile est inexistant, soit sur colluvium de pente, donc sur des matériaux mis en place très récemment, soit sur grès même en station horizontale.

Si dans certains cas les sols sur grès sont légèrement lessivés, la plupart du temps, le lessivage est inexistant. Deux exemples illustrent cette faible évolution des sols développés sur grès.

\subsection{Sol brun lessivé}

\section{Description}

0-10 $\mathrm{cm}$ horizon $\mathrm{A}_{1}$ : structure grumuleuse (couleur gris-rouge).

10-40 cm horizon $A_{2}$ : meuble, structure finement grumuleuse, excellent enracinement, 7,5 YR 6/4 (brun clair).

$40-80 \mathrm{~cm}$ horizon $\mathrm{B} \quad$ : moins meuble, structure massive, quelques racines $5 \mathrm{YR}$ $5 / 4$ (brun rouge).

80-et plus horizon $\mathrm{C} \quad$ : imperméable, grès non altéré.

Roche-mère : grès à voltzia " à faciès argileux \#

Position topographique : pente légère 1 à $2 \%$.

Les teneurs en fer libre et en argile des horizons $\mathrm{A}_{2}$ et $\mathrm{B}$, permettent de penser, qu'il y a effectivement eu lessivage. Les indices d'entrainement sont respectivement de 1,4 et 2 (Tableau 4). La teneur en fer libre passe nettement par un maximum aux environs de $60 \mathrm{~cm}$. Il en est de même pour la teneur en argile, qui passe par un maximum aux environs de $70 \mathrm{~cm}$. Cependant en $\mathrm{A}_{1}$, la teneur en argile est de $23,7 \%$ donc égale à celle de l'horizon $\mathrm{C}$. S'il y a eu lessivage comme le montre la variation de la teneur en fer libre, l'hétérogénéité de la roche-mère doit intervenir au même titre que le lessivage. A l'œil il est difficile de distinguer l'horizon B. Il est différent de la roche-mère, aussi bien par sa couleur, que par sa teneur en argile. II n'existe pas de structure polyédrique. Cette absence de structure polyédrique doit être due au faible taux d'argile $(27 \%$ en $\mathrm{B})$. On peut distinguer l'horizon $\mathrm{A}_{2}$ de l'horizon $\mathrm{B}$, à l'œil, en raison de la différence de structure, et d'une légère décoloration. Malgré cette faible différence du profil, ces sols développés sur grès ont été cartographiés comme sols bruns lessivés.

\subsection{Sol brun acide}

\section{Description}

$\mathrm{A}_{1} \quad$ : Moder - structure particulaire (gris foncé)

(B) : Structure particulaire - meuble - enracinement abondant 5 YR 5/6 (rouge jaunâtre). Transition diffuse entre les 2 horizons (B) et (B)/C.

(B)/C : Structure massive - compact 2,5 YR 4/4 (brun rouge) 
TABLEAU 4

Caractéristiques analytiques d'un sol brun lessivé sur grès à Voltzia

\begin{tabular}{|c|c|c|c|c|c|c|c|c|c|c|c|c|c|}
\hline & \multirow{2}{*}{$\begin{array}{l}\mathrm{pH} \\
\mathrm{cau}\end{array}$} & \multirow{2}{*}{$\begin{array}{c}\text { Matière } \\
\text { organique } \\
\% \\
\%\end{array}$} & \multirow{2}{*}{$\mathrm{C} / \mathrm{N}$} & \multicolumn{4}{|c|}{$\mathrm{me} / 100 \mathrm{~g}$} & \multirow{2}{*}{$\begin{array}{c}\text { Fe libre } \\
\text { (DEB) } \\
\%\end{array}$} & \multirow{2}{*}{ Argile } & \multirow{2}{*}{$\begin{array}{l}\text { Limons } \\
\text { fins }\end{array}$} & \multirow{2}{*}{$\begin{array}{c}\text { Limons } \\
\text { grossiers }\end{array}$} & \multirow{2}{*}{$\begin{array}{l}\text { Sables } \\
\text { fins }\end{array}$} & \multirow{2}{*}{$\begin{array}{l}\text { Sables } \\
\text { grossiers }\end{array}$} \\
\hline & & & & K & $\mathrm{Ca}$ & $\mathrm{Mg}$ & $\mathrm{T}$ & & & & & & \\
\hline$A_{1}$ & 4,7 & 12,7 & 20.5 & 1,06 & 2,78 & 1,67 & 16 & 0,79 & 23,7 & 24,1 & 13,7 & 20,7 & 2,4 \\
\hline $\mathrm{A}$ & 4,7 & 2,2 & 18,4 & 0.27 & 0.25 & 0,38 & 6 & 0,77 & 13,1 & 31,7 & 19.5 & 28,0 & 4,5 \\
\hline (B) & 4,8 & & & 0,27 & 0,35 & 0,64 & 6 & 1,08 & 27,2 & 20,8 & 11,6 & 36,0 & 2.7 \\
\hline C & & & & & & & & 1,00 & 23,3 & 17,1 & 9,0 & 46,1 & 2.9 \\
\hline
\end{tabular}

TABLEAU 5

Caractéristiques analytiques d'un sol brun acide sur grè̀s Vosgien

\begin{tabular}{|c|c|c|c|c|c|c|c|c|c|c|c|}
\hline & \multirow{2}{*}{$\begin{array}{l}\mathrm{pH} \\
\text { eau }\end{array}$} & \multirow{2}{*}{$\begin{array}{c}\text { Matière } \\
\text { organique } \\
\%\end{array}$} & \multirow{2}{*}{$\mathrm{C} / \mathrm{N}$} & \multicolumn{2}{|c|}{$\mathrm{me} / 100 \mathrm{~g}$} & \multirow{2}{*}{$\begin{array}{c}\text { Fe libre } \\
\text { (DEB) } \\
\%\end{array}$} & \multirow{2}{*}{ Argile } & \multirow{2}{*}{$\begin{array}{l}\text { Limons } \\
\text { fins }\end{array}$} & \multirow{2}{*}{$\begin{array}{l}\text { Limons } \\
\text { grossiers }\end{array}$} & \multirow{2}{*}{$\begin{array}{c}\text { Sables } \\
\text { fins }\end{array}$} & \multirow{2}{*}{$\begin{array}{c}\text { Subles } \\
\text { grossiers }\end{array}$} \\
\hline & & & & K & $\mathrm{Ca}$ & & & & & & \\
\hline$A_{1}$ & 3,8 & 9,2 & 22,3 & 0,35 & 0,34 & 0,61 & 12,3 & 15,2 & 7,1 & 10,7 & 43,8 \\
\hline (B) & 4,5 & 3,15 & 30,3 & 0.08 & 0,10 & 0.55 & 12,0 & 16,8 & 7,1 & 10,6 & 49,2 \\
\hline $\mathrm{B} / \mathrm{C}$ & 4,3 & & & 0,11 & 0.25 & 0,56 & 12,0 & 12,0 & 2,6 & 7,1 & 65,8 \\
\hline
\end{tabular}


Roche-mère : grès vosgien

Position topographique : pente nulle.

Il n'y a aucun entraînement du fer et de l'argile. L'horizon (B) ne se distingue de la roche-mère que par une coloration rougeâtre un peu moins marquée, et par sa moindre compacité.

\section{Discussion}

Il est particulièrement difficile de donner une explication à de telles différences d'évolution. En quelques mètres, on passe de sols très évolués, situés sur limons, à des sols peu évolués situés sur grès, donc sur un matériel à texture plus favorable à l'évolution, que celle des limons. Nous avons vu que les limons ont permis la formation de sols épais de $2,50 \mathrm{~m}$. Ces sols ultérieurement ont été érodés de 70 à $80 \mathrm{~cm}$. Cette hauteur correspond à peu près à la profondeur d'un sol normal. On peut supposer que sur grès, à l'époque où les limons étaient fortement lessivés, se sont développés des sols n'ayant pas plus de $1 \mathrm{~m}$ d'épaisseur, du fait de l'existence de la dalle de grès. L'érosion aurait ensuite enlevé 70 à $80 \mathrm{~cm}$ comme dans le cas des limons et aurait donc provoqué la disparition quasi totale de ces anciens sols. Après la reprise de la pédogenèse à l'époque récente, l'évolution serait repartie de néant sur les grès, ce qui expliquerait la faible évolution vers le sol brun lessivé ou les sols bruns acides. Le passage d'une roche-mère à l'autre correspond donc au passage brutal d'une période de pédogenèse ancienne à une période de pédogenèse récente, d'oủ la différence d'évolution. Les sols bruns acides et peut-être les sols bruns lessivés représenteraient donc, soit les sols climax, en équilibre avec la végétation forestière actuelle, soit une phase d'évolution initiale vers un stade plus évolué.

\section{6. - PODZOLS}

Dans ce massif existent des sols autres que les sols lessivés, ne représentant pas le climax. Ce sont des sols à hydromorphie permanente, dont la présence est liée à la topographie, et des sols de la série podzolique.

Des podzols typiques parfaitement différenciés n'existent que dans des zones à texture très grossière, là oủ les alluvions anciennes apparaissent dès la surface.

Si le substratum comprend des éléments fins limoneux ou argileux, même en faible proportion, il n'y a pas différenciation de podzols, même si la végétation est une pineraie à callune et myrtille.

Lorsque les podzols que nous avons cartographiés sont sous une végétation acidiphile de type pineraie, nous pouvons estimer qu'ils sont actuels, ou du moins, qu'ils se sont différenciés à une période récente.

Mais nous avons pu observer sur alluvions anciennes, et sous futaie de hêtres et de chênes des podzols bien différenciés à $\mathrm{A}_{2}$ atteignant 40 à $50 \mathrm{~cm}$.

II semble impossible qu'un tel sol ait pu se différencier sous un peuplement de feuillus. II semble plus logique dans ce cas de penser que cette podzolisation est l'œuvre d'une végétation actuellement disparue. 
Ces podzols sous feuillus pourraient done correspondre à une phase de pédogenèse beaucoup plus ancienne que celle à laquelle correspondent les podzols portant actuellement une pineraic.

\section{CONCLUSIONS GÊNÊRALES}

Par la cartographie à grande échelle d'un massif forestier des Basses-Vosges, nous avons été amenés à poser un certain nombre de problèmes de pédogenèse en raison de la complexité et de la diversité des sols.

Dans ce mémoire, nous avons émis un certain nombre d'hypothèses pour tenter de donner une explication à cet état de fait compliqué.

Ces hypothèses nous les avons étayées par des études détaillées de profils, en comparant ces profils entre eux, aussi bien par leurs caractéristiques morphologiques qu'analytiques.

Nous avons établi des graphiques de répartition du fer libre et de l'argile en fonction de la profondeur par analyses tous les $10 \mathrm{~cm}$.

Nous avons d'autre part, effectué les courbes cumulatives de 0 à $2000 \mu$ et de 2 à $2000 \mu$ pour divers horizons. Enfin, la comparaison des rapports $\frac{\text { fer total }}{\text { fer libre }}$ de différents sols et de dosage de l'aluminium libre, nous ont permis d'apporter quelques éléments supplémentaires.

Nous sommes ainsi arrivés à un schéma de la succession des différentes phases d'évolution des sols qui, bien que ne pouvant être qu'une approximation, doit cependant se rapprocher de la réalité.

Nous pouvons ainsi reconnaitre une première phase de rubéfaction intense, affectant les alluvions anciennes. Il semble que cette rubéfaction ne se soit pas produite en place, mais ait affecté le matériel avant son transport, probablement à la fin du pliocène ou au tout début du quaternaire.

Après le recouvrement de ces alluvions par une première couche de limons, nous pouvcns distinguer une nouvelle phase de rubéfaction intense ayant affecté la partie supérieure de ces limons. A cette phase correspondent des sols lessivés désaturés, ferrugineux à très faiblement ferralitiques, ou des ultisols si l'on adopte la classification américaine.

Cette phase serait à rapporter au quaternaire ancien.

Après l'érosion de la plus grande partie de ces limons anciens, rubéfiés, un nouveau placage de limons éoliens a été mis en place. Ces limons ont été soumis à une phase de lessivage intense, ayant abouti à la différenciation de sols lessivés de grand développement, dont le profil complet pouvait atteindre $2,50 \mathrm{~m}$.

Ce lessivage (indice d'entraînement du fer 2,5 à 2,7 - indice d'entraînement de l'argile 2,1 à 2,9 ) a provoqué l'imperméabilisation de l'horizon d'accumulation et l'apparition d'une nappe temporaire perchée. Des caractères d'hydromorphie sont venus se surimposer aux caractères hérités du lessivage. De sols lessivés, sensu stricto, nous sommes passés aux sols lessivés à pseudogley.

A cette phase de pédogenèse intense, que l'on peut situer avec beaucoup de 
prudence à l'interglaciaire Riss Würm, fait suite une phase d'érosion que l'on peut situer avec quelque vraisemblance, pendant ou à la fin de la dernière période froide. Cette érosion qui a pu atteindre, en certains endroits, 70 à $80 \mathrm{~cm}$ a eu pour conséquence de rapprocher plus ou moins de la surface, l'horizon de pseudogley. Elle a ainsi déterminé par sa plus ou moins grande amplitude la variation de l'intensité de l'hydromorphie actuellement constatée, ce qui conduit à classer les sols les plus érodés parmi les pseudogleys, et les moins érodés parmi les sols lessivés hydromorphes.

Après cette phase d'érosion la pédogenèse a repris sur des matériaux plus récents (limons remaniés ou colluvium) ou sur des matériaux mis à nu (grès à voltzia ou éventuellement alluvions anciennes).

Cette pédogenèse à laquelle on peut rapporter l'époque actuelle a abouti à la différenciation de sols peu évolués comparativement aux précédents, de type brun ou au plus brun lessivé, représentant le climax.

Sur matériel grossier comme les alluvions anciennes, il y a pu avoir, au cours de cette même période, différenciation de podzols que l'on peut assimiler à des sols azonaux. Certains de ces podzols peuvent se former actuellement sous végétation très acidiphile, d'autres, au contraire, situés sous feuillus, sont l'œuvre d'une végétation disparue.

Il semble donc, au moins dans le cadre de ce massif forestier que la différenciation des sols soit plus le résultat d'une évolution passée que celui de l'action des facteurs actuels.

Tant que cette évolution des périodes passées ne sera pas mieux connue, l'interprétation de l'aspect actuel des sols, à la lumière de modifications récentes, ne pourra être que très prudente.

Reçu pour publication en septembre 1967

\section{SUMMARY}

THE SOLLS OF A FOREST STAND IN THE \& LOWER-YOSGES $\$$. STAGES OF EVOLUTION

After surveying a large scale map of the soils (this map is not in the present publication), we tackled with some problems of soil genesis, especially of pseudogley soils, and with some problems of ancient evolution.

It appears that the present aspect of the soils results from a long evolution covering very different successive stages.

We can find out two stages of rubefaction. The former, dating probably from the end of the pliocene, affected the ancient alluvions before them being transported.

At the beginning of the quaternary, the latter affected ancient colian loams and permitted the differentiation of desaturated ferruginous (or very slightly ferallitic) soils.

We can then find out a stage of intense leaching which affected a layer of loams which are more recent than the former ones. The pseudogley leached soils date from that period which can be placed in all probability in the Riss Würm interglacial age. 
Effectively the leaching provoked the waterproofing of the B horizon ant the apparition of hydromorphic features, which superimposed themselves on the features resulting from the leaching.

A stage of an important erosion, which can be placed in the Würm or the end of the Würm, truncated then most of these soils. The nearer of the surface the pseudogley horizon is, the stronger the present hydromorphic features are, and so these features depend from the intensity of that ancient erosion.

Then the pedogenesis acted again upon ancient parent rocks (trias sandstones laid bare by this erosion), or upon some recently settled materials (remoulded loams and colluvial mellow deposits).

This new pedogenesis lead to far less developped soils : brown soils and leached brown soils.

\section{ZUSAMMENFASSUNG}

\section{DIE BÖDEN EINES WALDMASSIVS DER NIEDERVOGESEN}

DHE ENTWICKLUNGSSTADIEN

Nach der Aufnahme einer Bodenkarte im grossen Masstabe die hier nicht veröffentlicht wird. haben wir uos mit einige Fragen der Bodenbildung speziel, diejenige der Pseudogleyböden, und mit einige Problemen der frühere Bodenentwicklung befasst.

Es scheint dass die heutige Form der Böden das Ergebniss einer langen Entwicklung ist, die sich in Verschiedene Phasen einteilt.

Wir können zwei Rubifiezierungsphasen unterscheiden. Die erste, die warscheinlich aus dem Ende des Pliozenes stammt, hat sich auf die alten alluviale Ablagerungen, bevor ihrem Anschwemmen. ausgeübt.

Die zweite Anfangs des Quaternärs hat auf den alten äolischen Lehm gewirkt und hat die Unterscheidung von entbasten eisenhaltigen Böden oder sehr gering lateritischen Böden erlaubt.

Dann können wir eine intensive Tondurchschlämmungsphase unterscheiden die sich auf eine jüngere Lehmschichte als die vorhergehenden, ausgewirkt hat. Aus diesem Zeitalter, das man mit Wahrscheinlichkeit in die Zwischeneiszeit Riss-Würm situieren kann, stammen die Pseudogleyparabraunerden.

Die Tondurchschlämmung hat eine Underchlässigkeit des B-Horizontes und die Erscheinung der hydromorphen Merkmalen verursacht, die sich den Kennzeichen der Auswaszhung obendrein hinzugefügt haben.

Eine wichtige Erosionsphase die aus der Würm - oder Ende der Würmeiszeit stammt hat anschliessend diese Böden verstümmelt. Die heutige Hydromorphie ist um so grósser dass das Pseudogleyhorizont näher der Oberfläche liegt und ist also von der Stärke dieser alten Erosion abhängig.

Die Bodenbildung hat sich nun wieder auf älteren Grundgesteine (Sandsteine aus dem Trias) ausgeübt die durch die Erosion entblösst geworden sind oder auf Material das sich später Abgelagert hat (umgeinderter Lehm, und Kolluvium). Sie hat zu Böden geführt die Viel weniger entwickelt sind : Braunerde und ausgelaugte Braunerde.

\section{REFFÉRENCES BIBLIOGRAPHIQUES}

Ameryckx J., Wat.schot L., 1962. Note sur un ultisol fossile. Pédologie, 12, 2, 196-203.

A NDRÉ J., 1957. Les phẻnomẻnes de transports périglaciaires en Morvan Nord. Bull Ass. fr. Et. Sol., 91, $962-971$.

САсноN L., 1964. Contribution à l'étude du quaternaire récent de la Grande Limagne marno-calcaire. Ann, agron., 15-1, 59-68.

Dubors G., 1956. Modification des forêts et des climats dans les régions du Nord-Est et Sud-Est de la France. Actes du $4^{*}$ Congrès international sur le quaternaire Rome-Pise, 454-471.

Duchaufour Ph., 1948. Recherches écologiques sur la Chênaie Atlantique Française. Thèse. Ann. Ec, nation. Eaux Foréts, 11, I, 335.

Duchaufour Ph., 1957. Pédologie. Tableaux descriprifs et analytiques des sols, 87 p. Ec. nation. Eaux Forêts, Nancy. 
Duchaufour Ph., 1961. Données nouvelles sur la classification des sols. Ann. Ec, nation. Eaux Forêts, 18, 4, 9-67.

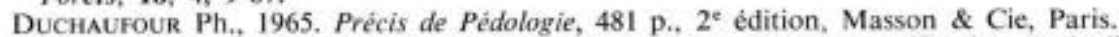

Duchaufour Ph et Souchitr B., 1965. Note sur un problème de classification. Podzolisation chimique et Différenciation du profil. Pédologie, 15, 2, 143-158.

Dupuis J., CAILleux A., 1955. Observations sur les formations superficielles et les sols de la Beauce au Sud d'Etampes. Ann. agron., Séric A, 373-383.

Edflman C.H., 1960. Podzols forestiers et podzols de bruyère. Pédologie, 10, 2, 229-249.

Franc de Ferrifre J., Millot G. et Camez Th., 1959. Argiles des sols des Formations tertiaires et quaternaires d'Aquitaine. Bull. Ass. fr. Et. sol, 2, 512-526.

GEZE B., 1959. La notion d'âge du sol. Son application à quelques exemples régionaux. Ann. agron., 3, 237-249.

Jackson M.L., 1965. Clay Transformations in Soil Genesis during the Quaternary, Soil S:i, 99, 1, 15-22.

JaMagne M., 1964. Introduction à une étude pédologique dans la partie nord du Bassin de Paris. Pédologie, 14, 2, 228-342.

LEFEVRE P., 1957. Les affleurements des formations géopédologiques picardes. Bull. Ass. fr. Et. Sol, $85,756-780$.

Le TACoN F., 1966. Contribution à P'etude des sols d'un massif forestier des Basses-Vosges. 121 p. Thèse Univ., Fac. Sci, Nancy.

MAIGNiEN R., 1961. Le passage des sols ferrugineux tropicaux aux sols ferrallitiques dans les régions Sud-Ouest du Sénégal. Sols afr., 6, 2 et 3, 113-173.

Mal.terre. H., 1946. Contribution à l'étude des limons quaternaires du Bassin de Paris. 108 p. Thèse, Fac. Sci, Paris.

Marocke R., 1956. Etudes pédologiques préliminaires des sols rouges et gris du Hardt. Ann. agron., 6, 889-893.

MAROCKE R., 1962. Les sols rouges paléoclimatiques de la terrasse würmienne rhénane alsacienne. C.R. Acad. Sci., 254, 3019-3021.

Marocke R., 1962. Datation des formations pédologiques fossiles et actuelles de la terrasse rhénane würmienne du Sud de I'Alsace. C.R. Acad. Sci., 254, 3116-3118.

Marocke. R., 1963-1964. Evolution des dépôts rhénans de la Haute-Alsace au cours du pleistocène récent. Bull. Soc. Hist. nat., Colmar, 51, 3-35.

Meriaux S., 1954. Contribution à l'étude de l'analyse gramulométrique. Thèse Fac. Sci. Dijon, 118 p.

Meriaux S., Gilly G., Lamontre B,, 1957. Sur le lessivage des sols de la plaine de la Saône. Bull. Ass. fr. Et. Sol, 83, 715-727.

Perigaud S., 1963. Contribution agronomique à la mise en valeur de la Brenne (sols hydromorphe3). Ann. agron., 14, 2 et 3, 121-331 et 261-377.

Plaisance G., 1966. Les sols à marbrure de la Forêt de chaux. Ann. Sci. forest., 22, 4, 437-680.

Tavernifr R, et Ameryckx J. Le postpodzol en Flandre sablonneuse. Pédologie, 7, 89-96.

Tricart J., 1956. Carte des phénomènes périglaciaires quaternaires en France. Mém. expl. de la carte géologique de France. Paris, 40 p.

Vanhoorne R., 1962, Datation des podzols fossiles dans les sables éoliens de Belgique, Pédologie, 12, 2, 154-158.

Vermeire R., 1962. Les sols de la Famenne entre Marche et Houyet. Pédologie, 12, 2. 204-236.

XXX, 1960. Soil classification. A comprehensive system, 7th approximation. Soil Survey stall, soll conservation service, USDA. 